\title{
Recent Strategies in Osteochondral Substitutes Design: Towards the Mimicking of a Multifaceted Anatomical Unit from the Nano to the Macro Level
}

Palazzo $B^{1,2 *}$, Scalera $F^{1}$, Soloperto $\mathbf{G t}^{3}$, Scialla $\mathbf{S}^{1}$ and Gervaso $F^{1}$

${ }^{1}$ Department of Engineering for Innovation, University of Salento, Campus Ecotekne, 73100, Lecce, Italy

${ }^{2}$ Ghimas S.p.A., c/o Dhitech S.c.a.r.l., via per Monteroni, Campus Ecotekne, 73100, Lecce, Italy

${ }^{3}$ Dhitech S.c.a.r.l, via per Monteroni, Campus Ecotekne, 73100, Lecce, Italy

\begin{abstract}
Nowadays regenerative medicine takes advantages not only of traditional implants, but also of engineered biocompatible parts, including degradable porous scaffolds integrated with cells or molecules.

These approaches, positioned at the interdisciplinary area between biomedical materials science and medicine, will become more achievable when combined with biomimicry, which not only optimizes biomaterial interaction with biological tissues but also mimics biogenic materials in their structure and functionalities.

Such methodologies are particularly attractive for bone and osteochondral related materials: in other words, cartilage and mineralized tissues are ideal examples to learn-from in setting up biomaterial science researches. These tissues are highly integrated systems, having found a compromise between different properties and functions, above all thanks to their hierarchical structure. Hard tissues display order on multiple lengths scale, from the nano to the macro level, whereas osteochondral tissue in particular exhibits an inherent gradient structure.

Bearing this in mind, bone and osteochondral scaffold design must enable hierarchical composites porous structures to attain desired mechanical function and mass transport, for example, permeability and diffusion, and to produce these structures within arbitrary and complex three-dimensional (3D) anatomical shapes.

In this context, this review will supply a hint of the strategies for biomimetic orthopaedic substitutes, actualizing the role of biomimicry, materials chemistry and process engineering in projecting and fabricating custom-made scaffolds.
\end{abstract}

Keywords: Regenerative medicine; Biomimicry; Hierarchy; Biomaterials; Scaffold; Osteochondral substitutes; Orthopaedic substitutes; Hydroxyapatite; Nanoceramics

\section{Introduction}

Nature is and will continue to be the best material scientist ever Nobody better than Nature can design composite constructions with complex organization and control the intricate phenomena that lead to the final shape and structure, from the macro to the nano-level, of living creatures. Nobody better than Nature can really design smart structural components that respond in situ to exterior stimuli, being able of adapting constantly their microstructure and correspondent properties.

Biomimicry studies how Nature designs, processes, and assembles molecular building blocks to fabricate high performance mineralpolymer composites (e.g. bone) and soft materials. These results should be ideally transferred into the fabrication of tissue grafts. Within such awareness, cartilage and mineralized tissues are ideal examples to learn-from in setting up biomaterial science researches.

As a matter of fact bone and chondral tissues are highly integrated systems, having found a compromise between different properties and functions. Such a high level of organization associates four aspects:

\section{Miniaturization}

Its object is to accommodate a maximum of elementary functions in a small volume

Hierarchy

Hierarchical constructions on a scale ranging from nanometres, micrometers, to millimetres are characteristic of biological structures and particularly of mineralized tissues introducing the capacity to answer the physical or chemical demands occurring at these different levels

\section{Hybridization}

The assembly of biological molecules or macromolecules and, in the case of bone, inorganic counterpart allows optimizing complementary possibilities and functions. In Nature, the hybridization process occurs at the nanoscale. Nature takes advantage of the higher strength and flaw tolerance of nanoscale inorganic building blocks to enhance the mechanical properties of a wide range of biological composites.

\section{Heterogeneity}

Natural composites are very heterogeneous "material systems" because they rely on local changes in chemical composition and structure at multiple length scales to change the local mechanical properties and thus meet the site-specific mechanical demands imposed by external stresses.

Bone has a hierarchical monolithic structure that is designed to

*Corresponding author: Barbara Palazzo, Department of Engineering fo Innovation, University of Salento, Italy, Tel: 00393391946966; E-mail: barbara.palazzo@unisalento.it

Received: August 16, 2017; Accepted: August 24, 2017; Published: August 30 2017

Citation: Palazzo B, Scalera F, Soloperto G, Scialla S, Gervaso F (2017) Recent Strategies in Osteochondral Substitutes Design: Towards the Mimicking of Multifaceted Anatomical Unit from the Nano to the Macro Level. J Nanomed Nanotechnol 8: 458. doi: 10.4172/2157-7439.1000458

Copyright: ( 2017 Palazzo B, et al. This is an open-access article distributed unde the terms of the Creative Commons Attribution License, which permits unrestricted use, distribution, and reproduction in any medium, provided the original author and source are credited. 
Citation: Palazzo B, Scalera F, Soloperto G, Scialla S, Gervaso F (2017) Recent Strategies in Osteochondral Substitutes Design: Towards the Mimicking of a Multifaceted Anatomical Unit from the Nano to the Macro Level. J Nanomed Nanotechnol 8: 458. doi: 10.4172/21577439.1000458

Page 2 of 7

reduce the inherent brittleness of the minerals, building either with fibres or with layers. Bone structure is based on very small building blocks in the nanometer scale, which may confer extraordinary defect tolerance to these composite. A three-dimensional (3D), hierarchical organization over many length-scales (nano-, micro-, millimetres and more) characterizes this particularly complex composite material.

The osteochondral unit is composed by two closely interconnected but very dissimilar tissues, the articular cartilage and the underlying subchondral bone. This crucial region can be defined a multifaceted structure in which articular cartilage is connected to the subchondral bone through an intermediated zone, i.e. the calcified cartilage. At the macro-scale, the chondral tissue is a hierarchical structure, strongly anisotropic, organized in multiple layers that differ each other in terms of components density and orientation.

Starting from these general considerations onto the bone and osteochondral tissues structures, this review describes examples of approaches of different scientists to supply bone and osteochondral tissues substitutes, able to mimic biological materials in their structure and functionality. A biomimetic and hierarchical approach will be followed also in the manuscript organization: initially, in the second section, biogenic ceramics synthesis and natural polymer guided assembly will be illustrated, subsequently, methods to process these buildings blocks into structure able to restore bone and osteochondral anatomical defects will be depicted in the third section.

\section{Approaches for Attaining Hierarchical Structures: Starting from Building Blocks}

It was recognized a long time ago that natural material and new material functionalities emerge from the assembly of nanoscale objects. Indeed, mechanical properties are largely determined at this length scale. The nanometer scale is also the one that living cells themselves spanning the microscale - control in an exquisite way, giving rise to elaborate materials with optimized designs, architectures, properties and functionalities. One of the underlying mechanisms that make nanomaterials superior to conventional materials for tissue engineering applications is that the former ones exhibit surface properties promoting protein adsorption. This process, favouring cell adhesion, Has a greater chance of stimulating new bone growth when compared to conventional materials [1].

As a consequence of these considerations, a nanotechnological approach is preferred in the field of bone and cartilage substitutes, both to achieve the required mechanical properties and to better resemble biological objects.

Far from being comprehensive, the following paragraph intends to elucidate some techniques aimed to obtain hybrids by a bottomup approach with the final goal of fabricating nanostructured bone and osteochondral scaffolds; in most cases, the assembly of objects at the nanoscale correspond to mimic the products of biological mineralization.

\section{Biological mineralization}

The bone organic matrix is mainly composed of type I collagen (90 wt.\%) that would act as template upon which the first mineral crystals were formed [2]. The not yet completely elucidated mechanism of cell-mediated collagen mineralization may be considered a sequence of events requiring the interaction of many different promoting or inhibiting factors [3]. It is widely accepted that matrix vesicles are formed by release of budding from osteoblasts surfaces [4-7], inside which the amount of calcium and phosphate raises to a saturation level, favourable for deposition of amorphous calcium phosphate, octacalcium phosphate and/or brushite with later transformation into HA [8]. The apatite nuclei from matrix vesicles act as templates for new crystal proliferation, which spread into the adjacent collagenous matrix [7]. Type I collagen is produced by osteoblasts, assembles within the cell to form triple helices and is then processed outside the cell. After excretion, the globular ends are cleaved off by enzymes and the 300 $\mathrm{nm}$ long triple-helical (apart from short telopeptide ends) molecules remain, ending in the surrounding extracellular space, where the fibrils organize theirselves into mature collagen fibrils that undergo bio-mineralization. For this reason, bone is the typical example of an "organic matrix-mediated" mineralization process [9].

Recently this mechanism has been further elucidated. In fact combining nanometre scale resolution cryogenic transmission electron microscopy and cryogenic electron tomography with molecular modelling, Nudelman and co-workers, in the Sommerdijk group, demonstrated that collagen functions in synergy with inhibitors of hydroxyapatite (HA) nucleation to actively control mineralization [10]. The positive net charge close to the C-terminal end of the collagen molecules promotes the infiltration of the fibrils with amorphous calcium phosphate (ACP). Furthermore, the clusters of charged amino acids, both in gap and overlap regions, form nucleation sites controlling the conversion of ACP into a parallel array of oriented apatite crystals. The banding pattern along the $67 \mathrm{~nm}$ repeat matched with the positions of the charged amino acids in the crystal structure of collagen [11,12]. After $24 \mathrm{~h}$ of mineralization, ACP was observed surrounding and entering the fibril. Moreover, the infiltration of mineral into the fibril through a particular band region is not dependent on the availability of space. Gaps within the microfibril are present throughout the whole $67 \mathrm{~nm}$ repeat, both in the gap and overlap regions, and could provide entry sites for the mineral phase into the fibril. Therefore, the sitespecific localization of mineral infiltration must result from a specific interaction between the amorphous mineral phase and the collagen at this location $[10,13]$.

\section{Bioinspired hybrid materials}

Mimicking bone and cartilage is a tremendous challenge and the aim for many in the fields of materials science and tissue engineering, especially since the design of such hybrid bio-organic/inorganic materials requires mineralization processes compatible with the organic components $[14,15]$.

This is particularly true in presence of collagen, the protein being irreversibly denaturized into gelatin through heating, then losing its self-ordering properties. Several groups have attempted to prepare bone-like materials from organic and mineral constituents, and hence someway trying to mimic biological mineralization. The leitmotiv of this strategy is the use of collagen $1 \mathrm{D}, 2 \mathrm{D}$ or $3 \mathrm{D}$ matrixes to act as template for mineralization

The in vitro self-assembly of collagen molecules induced by thermal or $\mathrm{pH}$ variation, to form native fibrils, illustrates that collagen molecules themselves contain all of the structural information necessary for the assembly [16]. Telopeptides-free type I collagen molecules have been utilized by Roveri and co-workers as a storehouse of information to nucleate carbonated HA nanocrystals inside the self-assembled collagen fibres [17]. The two components, HA nanocrystals and collagen fibrils, exhibit strong chemical and structural interactions that show a complete analogy of the synthesized composite with natural bone. The apatite crystals have nanometric dimensions, acicularshaped morphology, and preferential orientation of their c-axis. 
Citation: Palazzo B, Scalera F, Soloperto G, Scialla S, Gervaso F (2017) Recent Strategies in Osteochondral Substitutes Design: Towards the Mimicking of a Multifaceted Anatomical Unit from the Nano to the Macro Level. J Nanomed Nanotechnol 8: 458. doi: 10.4172/21577439.1000458

Page 3 of 7

In addition, bio-hybrids intended for metallic implant coating can be obtained through mineralization processes. Calcium phosphate/ collagen composites in fact, are limited to non-loading applications, but are particularly suitable to prepare bioactive coatings onto the surface of metallic implants, with the aim of accelerating bone formation and implant fixation. Electrolytic deposition of a biomimetic, bone-like self-assembled collagen fibrils/HA nano-crystals composite coating on titanium plates has been performed, using calcium $(\mathrm{Ca})$ and phosphorous $(\mathrm{P})$ precursors in a purely helical type I collagen molecule suspension (Figure 1a). The use of dilute electrolytic solutions, low current density at the cathode and room temperature affords a coating composed of poorly crystalline carbonate hydroxyapatite nano-crystals that nucleate inside and around the reconstituted collagen fibrils distributed in a homogeneous network on Ti plate surface [18].

Deshpande and Beniash [19] carried out bioinspired mineralization of reconstituted collagen fibrils using poly l-aspartic acid, as an analogue of non-collagenous acidic proteins. Transmission electron microscopy and electron diffraction studies of the reaction products revealed stacks of ribbon-shaped apatitic crystals, deposited within the fibrils with their c-axes co-aligned with the fibril axes. Initial mineral deposits formed in the fibrils lacked a long-range crystallographic order and transformed into crystals with time. Interestingly, the shape and organization of these amorphous deposits were similar to the crystals found in the mature mineralized fibrils. Authors demonstrated that the interactions between collagen and poly l-aspartic acid are essential for the mineralized collagen fibrils formation, while collagen alone does not affect mineral formation and poly l-aspartic acid inhibits mineralization in a concentration dependent manner.

Padmanabhan and co-workers [20] achieved the direct nucleation of $\mathrm{HA}$ on collagen fibres using calcium oxide $(\mathrm{CaO})$ derived from eggshells as $\mathrm{Ca}$ source and phosphoric acid as phosphate source. The obtained HA nanoparticles were embedded in the collagen and showed an elongated shape and size along the principal axis around $10 \mathrm{~nm}$. This original method, that takes advantage of a natural material as HA source, allowed to obtain composite scaffolds with an homogeneous, open and interconnected porous structure.

Most of these studies have successfully replicated an essential characteristic in bone, which is the predominant co-alignment of the organic and mineral phases. However, according to the Weiner and Wagner terminology [9], experiments have only been able to organize such structures up to the fibrillar level (range $=100 \div 300 \mathrm{~nm}$ ).

These aspects were highlighted by Nassif et al. [21], who, on the other hand, reported the preparation of a collagen-apatite matrix, enabling organization of collagen fibrils into 3D scaffolds and, concomitantly, allowing nucleation and co-alignment of HA crystals within the matrix from the nano- to millimetre scales. The process is based on a "one-pot" coprecipitation method at room temperature coupling the liquid-crystalline properties of collagen to a HA mineralization process. Indeed, the ability to organize collagen molecules at unusually high collagen concentrations through liquid crystalline ordered phase has been reported. The mineralized matrix reported by Nassif et al., has the characteristic multiscale bone-like hierarchy. Authors show that the dense and ordered collagen matrices together with a soft HA precipitation process are key factors for mimicking bone microarchitecture (Figures 1b-e). These results emphasize the role of physicochemical processes in biomineralization events that are most often discussed only from the viewpoint of biological control. The described mineral content of the collagen-apatite matrix is much lower than in fully developed bone. Nevertheless, bone development in vivo starts as a nearly unmineralized osteoid tissue that is gradually mineralized leading to a characteristic inhomogeneous mineralization pattern [22]. Thus, this bone mimetic matrix should also appear to be an appropriate substrate to study the behaviour of bone cells in vitro.

A further biomimetic approach, described in the papers by Altamura et al [23] consists in using a sol gel technique to synthesize HA nanocrystals inside a gelatin slurry. Briefly, a bicomponent system based on HA/gelatin was realized by an in situ mineralization technique inside the gelatin matrix, followed by a freeze-drying process. The combination of both processes led to a nanocomposite porous structure that was deeply characterized trough microdiffraction and microtomography, and discloses a gradient of both components. This case study allows introducing a recent principle in designing bioinspired hybrid materials.

It is interesting to see how bioinspired mineralization can be carried out along the direction of biomimetic osteochondral composite scaffolds resembling the composition of the extracellular matrices of cartilage and bone tissue. Tampieri and colleagues [24] implemented an example of this approach. Authors first aimed at further developing an above reported process of nucleation of hydroxyapatite nanocrystals onto self-assembled collagen fibres. Subsequently, they generated

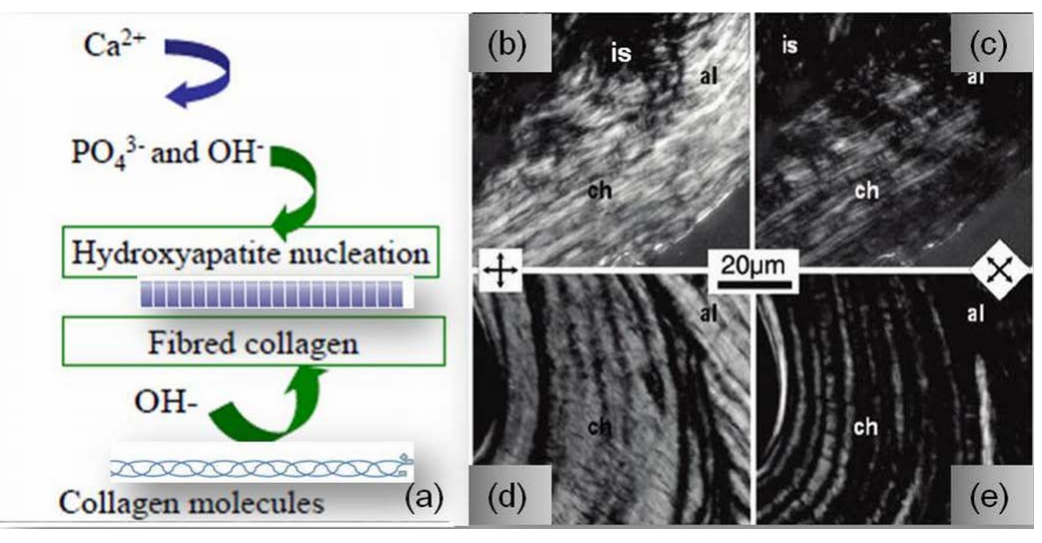

Figure 1: Scheme for the proposed mechanism for the electrochemically assisted deposition of a nanocomposite coating on a titanium surface (a). Thin section of the collagen apatite matrix obtained by Nassif et al. (b) and (c)) and comparison with bone matrix ((d) and (e)) [21] with permission, Copyright (2010) American Chemical Society). "Fibril array patterns" level ( 0.1-1 mm) is characterized by a spatial coexistence of different domains, i.e., isotrope (is), aligned (al), and twisted or cholesteric (ch). 
Citation: Palazzo B, Scalera F, Soloperto G, Scialla S, Gervaso F (2017) Recent Strategies in Osteochondral Substitutes Design: Towards the Mimicking of a Multifaceted Anatomical Unit from the Nano to the Macro Level. J Nanomed Nanotechnol 8: 458. doi: 10.4172/21577439.1000458

Page 4 of 7

chemically and morphologically graded hybrid materials, built by stacking a lower mineralized layer, produced according to the newly developed mineralization technique, an intermediate layer with reduced amount of mineral to mimic the tidemark (i.e. the calcifiednot calcified cartilage interface), and an upper layer formed by collagen and hyaluronic acid, reproducing some cartilaginous environmental cues. Please see the following section.

\section{Approaches for Attaining 3 D Structures and Complex Anatomical Shapes}

Continuing the bottom up approach that characterizes this review, after elucidating some strategies for the assemblage of organic and inorganic building block at the nanoscale, in the following paragraphs, methods for processing the above nano-materials will be described. Particularly, monolithic substitutes intended as bone like substitutes and multilayered scaffolds aiming to mimic the osteochondral tissue will be synthetically depicted.

\section{Bone-like scaffolds: bioceramic and nano-composite monolithic structures}

As in all tissue engineering strategies, the bone-like scaffolds must be highly porous with an interconnected 3D pore network for cell growth and transport of nutrients and removal of subsequent metabolic waste. Bone scaffolds should have suitable surface chemistry and topography for cell attachment, proliferation and differentiation, and their mechanical properties must be tailored to match those of the host tissues at the site of implantation. In order to achieve these requirements several fabrication techniques are currently used for bone scaffold production according to the material choice. The most used materials to this scope are, among the inorganic materials, ceramics and glasses, and among organic materials, various natural and synthetic polymers and composite of these. The analysis of the state of the art in the field reveals the presence of innovative techniques for scaffold and material manufacturing that are currently opening the way to the preparation of biomimetic substrates that modulate cell interaction for improved substitution, restoration, retention or enhancement of bone tissue function.

Describing all the above techniques is beyond the scope of this review. As a consequence of that, only some exemplificative methods will be proposed, probably the most widely used to process nanoceramics, natural polymers and the mineralized fibres described in Bionspired hybrid materials paragrah.

Among the bioceramics, HA is one the most used material because of its chemical similarity to the bone mineral [25]. Particularly, its role in improving biomineralization is a promising aspect for bone formation. A large number of studies reported the use of HA for producing bone scaffolds. The conventional method used to manufacture synthetic porous HA ceramics is by sintering a HA slurry mixed with organic polymer beads [26]. The polymer beads melt and vaporize during the sintering process, eventually leaving pores in the ceramic material. However, the pores resulting from this method are irregular in size and shape and not fully interconnected with one another. The sponge replica method, instead, is a quite simple method that allows for obtaining a very high and interconnected porosity using ceramic materials such as HA. As reported by Gervaso and colleagues [27] the preparation of HA scaffolds by polymeric sponge replica method allows for obtaining high-resistance interconnected macro-channeled porous scaffolds with over $500 \mu \mathrm{m}$ sized pores and $90 \%$ porosity that evidence superior mechanical properties with respect to those reported in literature.
In the same way in which HA is one of the mainly used bioceramics, collagen is one of the most used natural polymer, being one of the major component of the connective tissues. Collagen scaffolds are reported to promote a biological response, such as cell interaction, and to work as artificial biomimetic extracellular matrices that guide tissue regeneration.

A number of fabrication technologies have been applied to process collagen and other biodegradable and bioresorbable materials into 3D polymeric scaffolds with high porosity and surface area, including freeze drying, fibre bonding, electrospinning, solvent casting, particulate leaching, membrane lamination and melt molding [28]. Among them, it is worth citing electrospinning that allows obtaining biomimetic fibrous synthetic biomaterials. A polymer or a polymer containing suspended nanoparticles is dissolved in a solvent and pumped to the tip of a needle, which is separated from an earthed metal collecting plate. A high voltage potential difference (typically $20-40 \mathrm{kV}$ and at a few $\mathrm{mA}$ ) is applied between the needle and the plate. A fine polymer fibre is drawn with from the needle tip and the solvent evaporates en-route. A mat of spun fibres is thus gradually built at the collection plate point and it can then be peeled away from the plate. However this technique is more affordable for obtaining two-dimensional (2D) scaffolds and cartilaginous layers rather than $3 \mathrm{D}$ bone scaffolds.

Having mentioned the electrospinning, we will limit our description to the phase separation and lyophilisation technique, also known as freeze-drying. The reason for this choice, is not only because freeze drying is the most frequently used method for the fabrication of polymeric scaffolds, but also because it allows processing of mineralized polymers, towards bone intended nanocomposites.

According to freeze-drying technique, a polymer solution is prepared, introduced into a mould of interest and subjected to controlled freezing. Upon freezing, ice crystals form in the polymer solution, and are phase-separated from the polymer. During the subsequent freeze-drying step, the ice crystals sublimate to yield a porous structure of solid polymeric material. The scaffold pore structure, in terms of pore diameter, orientation and interconnectivity, varies depending on multiple processing parameters including freezing temperature, thermal gradients, and polymer concentration. In a similar way, suspensions of mineralized polymer or nanoHA (nHA) functionalized collagen fibres can be processed towards bone intended nanocomposites. We highlighted that, the current trend to replicate the extraordinary strength and durability of natural bone is achieved by designing biomaterials that nearly mimic the structural organization of bone from the nanoscale upward. In recent years, there has been an effort to join two or even more techniques to produce bimodal scaffolds, where micro- and nanoscale features can be combined. Among the above-cited techniques, freeze-drying and electrospinning can be linked toward the fabrication of 3D nano and microstructured scaffolds. For a thick 3D block structure, the electrospun fibers may either be evenly distributed throughout the freeze-dried structure or fit in as distinct layers. Vaquette and Cooper-White (2013) demonstrated the possibility of fabricating a multi-layered scaffold by stacking polycaprolactone (PCL) electrospun fibrous membrane into a holder filled with poly(lactic-co-glycolic acid) (PLGA) solution. They used quenching in liquid nitrogen followed by leaching in cold water to remove the solvent such that PLGA acts as binders. Based on the same concept freeze drying may also be carried out to form porous interlayers between the fibrous layers [29].

\section{Osteochondral like scaffolds: multi-layered structures}

While the above-described conventional monophasic scaffolds 
Citation: Palazzo B, Scalera F, Soloperto G, Scialla S, Gervaso F (2017) Recent Strategies in Osteochondral Substitutes Design: Towards the Mimicking of a Multifaceted Anatomical Unit from the Nano to the Macro Level. J Nanomed Nanotechnol 8: 458. doi: 10.4172/21577439.1000458

Page 5 of 7

result adequate to replace bone defects, it is clear that they cannot replace defective interfacial cartilage-to-bone tissue that possesses anisotropic structural properties and functions. Since the multifaceted organization of the ECM of the osteochondral unit is fundamental to grant functionality of articular cartilage, one of the main challenges for cartilage tissue engineering remains the high fidelity reproduction of all layers with proper orientation of collagen fibres and, consequently, suitable mechanical properties [30].

In order to regenerate such a complex structure in which the two main tissues, bone and cartilage, show extremely different morphological compositions and mechanical properties, biphasic or triphasic constructs have been developed. Engineering multilayer scaffolds with controlled properties in each layer could allow the replication of the local microenvironment of the osteochondral tissue. For satisfying the biological requirements, an osteochondral implant should ideally have a rigid osseous layer (to support the overlying cartilage and integrate with the native bone) and a chondral layer (to enable the seeding and proliferation of chondrocytes or mesenchymal stem cells and subsequent deposition of cartilaginous extracellular matrix) [31]. Moreover, the integrated materials can transfer physical or chemical stimuli from the cartilage to the bone layers, because the interface tissue is exposed to shear forces over a large range of motion.

Therefore, a successful procedure for the production of an osteochondral substitute, should consider the design of a bilayer structure in which the two layers are aimed to regenerate simultaneously bone and cartilage. Different strategies have been proposed by several authors in the recent years [32-34]. Most of the procedures consist of producing the two layers separately and connecting them secondarily. This strategy allows for the in vitro culture of each layer with suitable cell population. However, in the perspective of producing a cellfree osteochondral substitute emulating the complex osteochondral structure, bilayered scaffolds, designed to mimic each tissue type independently, may be more convenient than monolithic constructs with different functional requirements of both bone and cartilage in a single structure. Such bilayered scaffolds are formed in a single composite construct before implantation. Instead of cell seeded scaffolds, where neocartilage is generated by seeding chondrocytes on a support to form the cartilaginous layer independently from the bonny support, multilayer structures are designed to repair osteochondral defects by using tailored bilayered composite structure, which mimic the structure of articular cartilage and subchondral bone tissue. The integration at the interface between engineered cartilage and the subchondral bone part is developed via fabrication methods to form the physical integration before implantation.

Gotterbam et al. [35] developed a two-layered implant consisting of a basal porous beta-tricalcium phosphate for bone reconstruction and a superficial fibrous type I/III collagen layer for cartilage regeneration. The bilayer substitutes were implanted on minipigs and compared with both untreated lesions and grow factor-augmented implants. Such additional growth factor mixture was assumed to stimulate cell and tissue differentiation. The tissue regeneration was evaluated after 6,12 and 52 weeks. Treatment with the two-layered implant improved defect filling and subchondral bone repair at 6 and 12 weeks followup. After 52 weeks, the beta-tricalcium phosphate was replaced by cancellous bone. However, cartilage tissue repair consisted mainly of fibrocartilage and showed a moderate cell density. The addition of growth factor improved the mechanical and morphological properties of the cartilage repair tissue at 12 , but not at 52 weeks.

Few scaffolds, among those developed through research efforts, have reached the market. A successful example was the TruFit Plug (Smith \& Nephew, San Antonio, TX). TruFit Plug is a synthetic acellular two phases scaffold made from a polylactide-coglycolide copolymer that has been used as a treatment method for primary osteochondral defects or for gap filling of donor sites during OATS (osteochondral autograft transfer system) procedures. The bone phase contains calcium sulphate for stimulation of bone formation. Cartilage regeneration should be instigated by the integration of cells and growth factors derived from the bone marrow that infiltrates the plug. Williams and Gamradt [36] examined the efficacy of this scaffold in the femoral condyles and trochleae of goats and showed good filling of osteochondral defects, good integration in the native cartilage and a high percentage of hyaline-like cartilage and good bony restoration. Nevertheless, a very recent systematic review of clinical studies concerning the TruFit Plug [37] assessed that the available data do not support superiority of the TruFit plug in terms of clinical improvement compared to conservative treatment or other cartilage techniques. The aim of this biphasic scaffold is to regenerate both hyaline cartilage formation and subchondral bone ingrowth, but conflicting evidence exists on the properties of the newly formed cartilage, and none of the studies could provide evidence for osteoconductive bone ingrowth.

Another example of cell-free multi-layered scaffold was proposed by Tampieri and colleagues [22,38] that developed a composite osteochondral scaffold, organized in different integrated layers, mimicking articular cartilage and subchondral bone and intended to differentially support the formation of such tissues. Such substitute consists of a graded structure including a lower layer of biomineralized collagen, corresponding to the subchondral bone, an upper layer of hyaluronic acid-charged collagen, reproducing the cartilaginous region, and an intermediate layer similar to the biomineralized collagen, but with a lower mineral content, resembling the tidemark. The three layers are stacked and freeze-dried to obtain an integrated monolithic composite (Figure 2a-2c). The above-described graded scaffold, after the required evaluation, is now commercialized named as MaioRegen ${ }^{\otimes}$ and used in clinics. Very recent studies reported clinical outcome of patients treated with MaioRegen ${ }^{\circ}$ at a minimum followup of 2 years. Delcogliano and colleagues [39] reported a significant improvement of the evaluated clinical parameters (International Repair Cartilage Society score, the Tegner Score and EQ-VAS). The significant clinical improvements were confirmed by Christinseen et al. [40] that however reported an incomplete cartilage repair and poor subchondral bone repair at 1- and 2.5-year follow-up by treatment of osteochondral defects in the ankle and knee joint with the graded biomimetic scaffold MaioRegen ${ }^{\circ}$.

As another example, a novel 3D bicomponent substitute made of type I collagen and HA was developed $[27,41]$ and tested for the repair of osteochondral lesions in a swine model [42]. This scaffold was assembled by a newly developed method that guarantees the strict integration between the organic and the inorganic parts, mimicking the biological tissue between the chondral and the osseous phase (Figure 2d).

The in vivo study showed that the novel osteochondral scaffold was easy to handle for surgical implantation, was stable within the site of lesion and, at the end of the experimental time, well integrated with the surrounding tissue without any signs of synovitis. The quality of the reparative tissue indicated the promising potential of this novel biomaterial for use in a one-stage procedure for osteochondral repair.

In order to fabricate complex shaped, custom-made scaffolds, some of the cited techniques may be combined with digital tools such 
Citation: Palazzo B, Scalera F, Soloperto G, Scialla S, Gervaso F (2017) Recent Strategies in Osteochondral Substitutes Design: Towards the Mimicking of a Multifaceted Anatomical Unit from the Nano to the Macro Level. J Nanomed Nanotechnol 8: 458. doi: 10.4172/21577439.1000458

Page 6 of 7
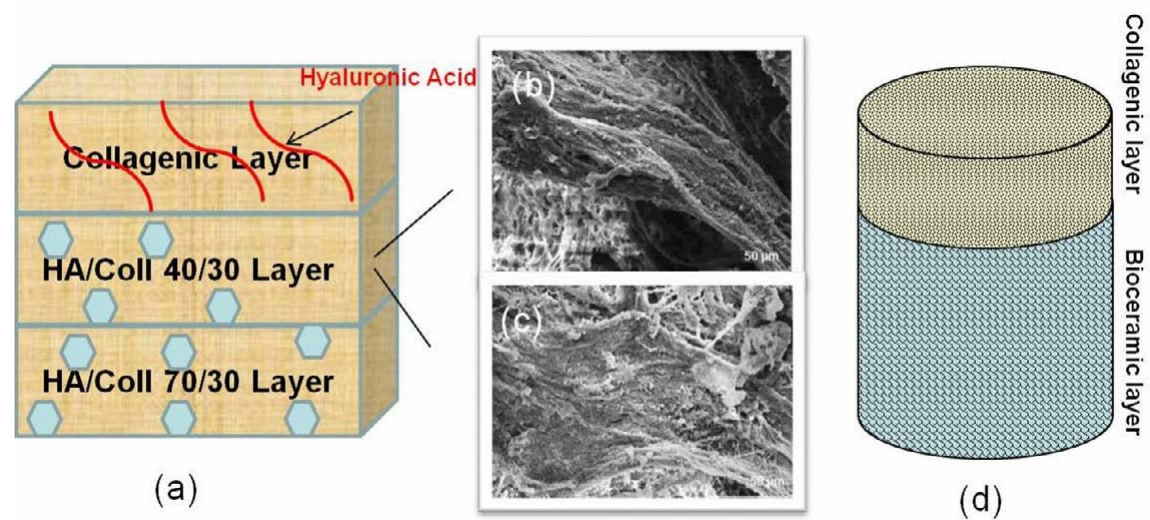

(d)

Figure 2: Scheme of osteochondral scaffold proposed by Tampieri et al. [24] (a). The mineralized bony layer is well noticeable in the SEM image (b) and (c), reproduced with permission [38], copyright (2015) Elsevier. Schema for the osteochondral scaffold proposed by Gervaso et al. [27] (d).

as 3D Computer Aided Design (CAD) systems and rapid prototyping (RP) machine. Particularly, RP techniques allow the construction of complex physical models based on $3 \mathrm{D}$ clinical images elaborated by suitable software and CAD systems [43]. Besides projecting complex shape, RP machine can also be used to produce complex moulds allowing obtaining scaffolds with improved mechanical and anatomical performances [44]. Gervaso and co-workers for example projected a new intended scaffold aimed to support the axial loading and contemporarily to undergo deformation in the radial direction (Figure 3). Specifically, a set thin columnar elements, made of porous HA, constitute the reinforcing units of the collagen matrix. This set constitutes the bony part and is towered over by a pure collagen layer (the chondral layer so called "honeycomb-shaped" scaffold) [44]. In this case the RP technique has been used to print the mould in which collagen gel has been freeze-dried for obtaining the complex-shaped polymer matrix. On the other hand, the replica sponge method allowed producing the thin columns.

Moreover, it is worth noting that the resorbibility of the ceramic components in the "honey-comb shaped "scaffold can be significantly incr eased with respect to that one of the pure HA. On the one hand, it is possible to develop magnesium-doped apatites that mimic the composition of mineral bone with the aim of enhancing their bioactivity and increasing bone regeneration. On the other hand, a feasible approach is also the use of composites in which one reinforcing phase with superior osteoconductive properties- such as calcium silicates, and particularly wollastonite $\left(\mathrm{CaSiO}_{3}\right)$ is added to a $\mathrm{HA}$ matrix [45]. Both Mg-substituted HA and HA/Wollastonite composites have been used as starting materials for the production of the thin columnar elements of the "honeycomb-shaped" scaffolds [45]. The abovedescribed substitutes have been tested in a critical osteochondral lesion generated in the medial femoral condyle of 18 skeletally mature sheep. Animals were sacrificed after 3 months and samples were analysed by computer tomography (CT), magnetic resonance imaging (MRI), macroscopic evaluation and histology. The study demonstrated that the scaffold with the columnar elements made of Mg-substituted HA possesses the potential for being applied for one-stage procedures for osteochondral defects [46].

Although biphasic materials consisting of a cartilage and a bone part have been described and are still being developed by several authors, it has to be underlined that many other anatomical aspects at the nanoscales of both these tissues combined in the osteochondral unit should be reproduced.

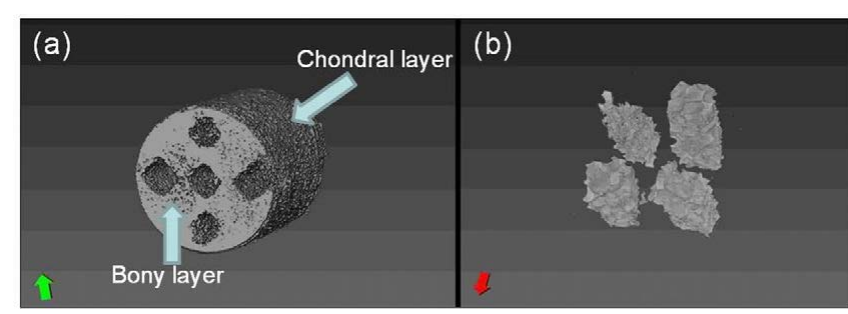

Figure 3: MicroCT of the "honeycomb shaped" scaffold proposed by Gervaso and colleagues. Collagenic matrix (a) contains the loci for hosting porous HA columns (b) Bioceramic columns aim both to axially reinforce the polymer matrix and both to supply the surface for bone cells adhesion. Courtesy of Nacucchi, ENEA research centre of Brindisi.

\section{Summary and Outlook}

This review highlighted how biological materials exhibit unique control over the local composition, concentration and orientation of building blocks at multiple length scales to best respond to the typically non-uniform mechanical load imposed by their natural environment.

As a consequence of it, extending the biological concept of spatially tuneable architectures to biomedical applications should lead to advanced graded composites with response tailored to optimize specific targeted functions. Bearing this in mind, we underlined approaches for assembling organic and inorganic building blocks at the nanoscale and further to process them up to obtain $3 \mathrm{D}$ and sometimes complex shaped scaffolds. We gave examples of biphasic materials consisting of a single cartilage and bone part, some of which attempting to mimic natural heterogeneity and hierarchy of biological tissues.

Anyway, despite the excellent research in this field, it has to be noted that no artificial materials has been able to replicate bone and above all cartilage properties. Moreover, examples of scaffold for bone tissue engineering which are suitable to be translated from the lab bench to the market are still limited. All these aspects leave many open questions, one of them probably being which level of complexity should reach the system scaffold to succeed.

In conclusion it is definitively crucial for an excellent material scientist in the tissue engineering field not to leave appropriate surgical techniques out of consideration and, as a matter of fact, inspired design, technical innovation and precise craftsmanship should be joined with biologists and clinicians advices in order to reach the hoped results. 
Citation: Palazzo B, Scalera F, Soloperto G, Scialla S, Gervaso F (2017) Recent Strategies in Osteochondral Substitutes Design: Towards the Mimicking of a Multifaceted Anatomical Unit from the Nano to the Macro Level. J Nanomed Nanotechnol 8: 458. doi: 10.4172/21577439.1000458

\section{Acknowledgments}

We are grateful to Prof. Sannino (University of Salento) and Prof. Peretti (Istituto Ortopedico Galeazzi, Milan) for fruitful discussions.

\section{References}

1. Gautrot JE, Malmstrom J, Sundh M, Margadant C, Sonnenberg A, et al. (2014) The nanoscale geometrical maturation of focal adhesions controls stem cell differentiation and mechanotransduction. Nano Lett 14: 3945-3952.

2. Weiner S, Wagner HD (1998) The Material bone: structure-mechanical function relations. Ann Rev Mat Sci 28: 271-298.

3. Sikavitsas VL, Temenoff JS, Mikos AG (2001) Biomaterials and bone mechanotransduction. Biomaterials 22: 2581-2593.

4. Anderson HC (1984) Mineralization by matrix vesicles. Scan Electron Microsc pp: 953-964.

5. Posner AS (1985) The mineral of bone. Clin Orthop 200: 87-99.

6. Rodan GA (1992) Introduction to bone biology. Bone 13: 53-56.

7. Anderson HC (1995) Molecular biology of matrix vesicles. Clin Ortop 314: 266-280

8. Anderson HC (2003) Matrix vesicles and calcification. Curr Rheumatol Rep 5 222-226

9. Lowestan HA, Weiner S (1989) On biomineralization. Oxford University Press, New York, USA.

10. Nudelman F, Pieterse K, George A, Bomans PHH, Friedrich H, et al. (2010) The role of collagen in bone apatite formation in the presence of hydroxyapatite nucleation inhibitors. Nat Mater 9: 1004-1009.

11. Chapman JA, Tzaphlidou M, Meek KM, Kadler KE (1990) The collagen fibril - A model system for studying the staining and fixation of a protein. Electron Microsc Rev 3: 143-182.

12. Orgel JPRO, Irving TC, Miller A, Wess TJ (2006) Microfibrillar structure of type I collagen in situ. Proc Natl Acad Sci USA 103: 9001-9005.

13. Palazzo B, Foltran I, Walsh D (2012) Morphological surface characterization of nano-structured inorganic and polymeric materials for biomedical application. In: Rimondini (ed.) Bentham Publisher, Sharjah (United Arab Emirates).

14. Palazzo B, Gervaso F, Scalera F, Casillo A, Ambrosio L, et al. (2014) Biohybrid scaffolds for bone tissue regeneration: a critical comparison between hydroxyapatite/agarose and hydroxyapatite/chitosan nano-composites. Journal of Tissue Engineering and Regenerative Medicine.

15. Palazzo B, Izzo D, Scalera F, Cancelli AN, Gervaso F (2015) Bio-hybrid scaffolds for bone tissue engineering: nano-hydroxyapatite/chitosan composites. Bioceramics 631: 300-305.

16. Falini G, Fermani S, Foresti E, Parma B, Rubini K, et al. (2004) Films of selfassembled purely helical type I collagen molecules. J Mater Chem 14: 2297-2302.

17. Roveri N, Falini G, Sidoti MC, Tampieri A, Landi E, et al. (2003) Biologically inspired growth of hydroxyapatite nanocrystals inside self-assembled collagen fibers. Mater Sci Eng 23: 441-446.

18. Manara S, Paolucci F, Palazzo B, Marcaccio M, Foresti E, et al. (2008) Biomimetic hydroxyapatite-collagen coating electrochemically deposited on titanium plate. Inorganica Chimica Acta 361: 1634-1645.

19. Deshpande AS, Beniash E (2008) Bio-inspired Synthesis of Mineralized Collagen Fibrils. Cryst Growth Des 8: 3084-3090.

20. Padmanabhan S, Salvatore L, Gervaso F, Catalano M, Taurino A, et al. (2014) Synthesis and Characterization of Collagen Scaffolds Reinforced by Eggshell Derived Hydroxyapatite for Tissue Engineering. Journal of Nanoscience and Nanotechnology 14: 1-6.

21. Nassif N, Gobeaux F, Seto J, Belamie E, Davidson P, et al. (2010) SelfAssembled Collagen-Apatite Matrix with Bone-like Hierarchy. Chem. Mater 22 3307-3309.

22. Roschger $P$, Paschalis EP, Fratzl P, Klaushofer K (2008) Bone mineralization density distribution in health and disease Bone 42: 456-66.

23. Altamura D, Pastore SG, Raucci MG, Siliqi D, De Pascalis F, et al. (2016) Scanning Small- and Wide-Angle X-ray Scattering Microscopy Selectively Probes HA Content in Gelatin/Hydroxyapatite Scaffolds for Osteochondral Defect Repair ACS Appl. Mater Interfaces 8: 8728-8736.
24. Tampieri A, Sandri M, Landi E, Pressato D, Francioli S, et al. (2008) Design of graded biomimetic osteochondral composite scaffolds. Biomaterials 9: 3539-3546.

25. Le Geros RZ, Le Geros JP (1985) Phosphate Minerals, Nriagu JO, Moore PB (eds.) Springer Publisher, New York, USA.

26. Uchida A, Nade SM, McCartney ER, Ching W (1984) The use of ceramics for bone replacement. A comparative study of three different porous ceramics. J Bone Joint Surg Br 66: 269-275.

27. Gervaso F, Scalera F, Kunjalukkal Padmanabhan S, Licciulli A, Deponti D, et al. (2012) Development and Mechanical Characterization of a Collagen/ Hydroxyapatite Bilayered Scaffold for Ostechondral Defect Replacement. Key Engineering Materials 493-494: 890-895.

28. Hutmacher DW (2000) Scaffolds in tissue engineering bone and cartilage Biomaterials 21: 2529-2543.

29. Vaquette C, Cooper-White J (2013) A simple method for fabricating 3-D multilayered composite scaffolds. Acta Biomaterialia 9: 4599-4606.

30. Martin I, Miot S, Barbero A, Jacob M, Wendt D (2007) Osteochondral tissue engineering. J Biomech 40: 750-65.

31. Shimomura K, Moriguchi Y, Murawski, CD, Yoshikawa H, Nakamura N (2014) Osteochondral Tissue Engineering with Biphasic Scaffold: Current Strategies and Techniques. Tissue Engineering: Part B 20: 468-476.

32. Mano JF, Reis RL (2007) Osteochondral defects: present situation and tissue engineering approaches. J Tissue Eng Regen Med 1: 261-273.

33. O'Shea TM, Miao X (2008) Bilayered scaffolds for osteochondral tissue engineering. Tissue Eng: Part B 14: 447-464.

34. Nooeaid P, Salih V, Beier JP, Boccaccini AR (2012) Osteochondral tissue engineering: scaffolds, stem cells and applications. J Cell Mol Med 16: 2247-2270.

35. Gotterbarm T, Richter W, Jung M, Berardi Vilei S, Mainil-Varlet $P$, et al. (2006) An in vivo study of a growth-factor enhanced, cell free, two-layered collagentricalcium phosphate in deep osteochondral defects. Biomaterials 27: 3387-3395.

36. Williams RJ, Gamradt SC (2008) Articular cartilage repair using a resorbable matrix scaffold. Instr Course Lect. 57: 563-571.

37. Verhaegen J, Clockaerts S, Van Osch GJV, Somville J, Verdonk P, et al. (2015) TruFit Plug for Repair of Osteochondral Defects-Where Is the Evidence? Systematic Review of Literature. Cartilage 6: 12-19.

38. Minardi S, Corradetti B, Taraballi F, Sandri M, Van Eps J, et al. (2015) Evaluation of the osteoinductive potential of a bio-inspired scaffold mimicking the osteogenic niche for bone augmentation. Biomaterials 62:128-37.

39. Delcogliano M, de C aro F, Scaravella E, Ziveri G, De Biase CF, et al. (2014) Use of innovative biomimetic scaffold in the treatment for large osteochondral lesions of the knee. Knee Surg Sports Traumatol Arthrosc 22: 1260-1269.

40. Christensen BB, Foldager CB, Jensen J, Jensen NC, Lind M (2015) Poor osteochondral repair by a biomimetic collagen scaffold: 1 - to 3-year clinical and radiological follow-up. Knee Surg Sports Traumatol Arthrosc 22: 1260-1269.

41. Deponti D, Di Giancamillo A, Gervaso F, Domenicucci M, Domeneghini C, et al. (2014) Collagen scaffold for cartilage tissue engineering: the benefit of fibrin glue and the proper culture time in an infant cartilage model. Tissue Eng Part A 20: 1113-1126.

42. Sosio C, Di Giancamillo A, Deponti D, Gervaso F, Scalera F, et al. (2015) Osteochondral repair by a novel interconnecting collagen-hydroxyapatite substitute: a large-animal study. Tissue Eng Part A 21: 704-15.

43. Gervaso F, Scalera F, Licciulli A, Sannino A (inventors) EP2481554 (A1) Method for producing bone substitutes and/or fillers made to measure and made of bioactive and biomimetic materials. Applicants: Ghimas Spa.

44. Peretti G, Fraschini G, Sannino A, Gervaso F, Scalera F, et al. (Inventors) W02014184391 (A1) Composite scaffold for tissue repair. Applicants: Ospedale San Raffaele Srl; Università Del Salento.

45. Izzo D, Scalera F, Palazzo B, Cancelli AN, Scialla S, et al. (2016) "HONEY": a radially-compliant scaffold for osteochondral defects of a critical size". Proceeddings of the 10th World Biomaterials Congress (WBC 2016) Montreal QC (Canada), Frontiers in Bioengineering and Biotechnology.

46. Gervaso F, Mangiavini L, Di Giancamillo A, Boschetti F, Izzo D, et al. (2016) Comparison of three novel biphasic scaffolds for one-stage treatment of osteochondral defects in a sheep model. J Biol Regul Homeost Agents 30: 24-31. 